REVIEW ARTICLE

\title{
The Role of JAK2 in Myeloproliferative Diseases Diagnosis
}

\author{
Yudith Annisa Ayu Rezkitha ${ }^{1 *}$
}

${ }^{1}$ Department of Internal Medicine, Faculty of Medicine Universitas Airlangga - Dr. Soetomo General Hospital Surabaya, Indonesia

\section{A R T I C L E I N F O}

\section{Article history:}

Received 25 September 2018

Received in revised form 15

October 2018

Accepted 1 November 2018

Available online 30 November 2018

Keywords:

Janus Kinase 2,

Myeloproliferative disease,

Mutation.

*) Corresponding author:

yudithannisaayu@gmail.com

\begin{abstract}
A B S T R A C T
Janus Kinase 2 (JAK2) plays an important role in mediating transduction signal of hematopoiesis, including in the pathogenesis of Myeloproliferative diseases (MPD). Various studies have been carried out to identify the position of aleles in tyrosine encoding mutations. Although the effect of JAK2 mutations is still not fully understood, the discovery of these mutations might be able to differentiate the types of polycythaemia vera, essential thrombocytemia, and primary myelofibrosis with malignant abnormalities. WHO has revised the MPD diagnosis criteria following this finding. This review will discuss the role of JAK2.
\end{abstract}

\section{Introduction}

Myeloproliferative diseases (MPD) are clonal abnormalities of hematopoiesis progenitors. This group consists of two main groups, namely the Philadelphia positive chromosome group Chronic Myeloid Leukemia (CML), the negative Philadelphia chromosome group namely Polycythemia Vera (PV), Essential Thrombocytemia (ET) and Primary Myelofibrosis (PMF), as well as the rare MPD group such as Chronic Eosinophilic Leukemia (CEL), Chronic Myelomonocytic Leukemia (CMML), and Systemic Mastocytosis (SM). ${ }^{1}$

Janus Kinase 2 (JAK2) is a part of tyrosine kinase that is important for cytokine receptors and plays a role in mediating signal transduction from various immune responses, growth, cell differentiation, and hematopoiesis. It is now known that mutations in JAK2 will cause constitutive activation of the JAK pathway. Various studies have been conducted to identify the position of the activated alele, causing a constitutive abnormality in tyrosine kinase encoding. The discovery of the JAK2V617F mutation provides a way to differentiate polycythaemia vera, ET and PMF with benign and malignant abnormalities that are clinically similar, although they cannot distinguish between them. ${ }^{2}$

Although the latest information about the effect of JAK2 mutations on the pathogenesis and prognosis of MPD is still not entirely clear, various evidences about JAK2 has made the World Health Organization (WHO) revise the criteria for MPD diagnosis. ${ }^{3}$

\section{JAK2}

The human body is able to express more than 500 protein kinases. There are 2 types of tyrosine type protein kinases namely receptor and cytoplasm types. Protein tyrosine kinase receptor types include Platelet-Derived Growth Factor Receptors (PDGFR), stem cell factor receptor (KIT), Fibroblast Growth Factor Receptor (FGFR), Vascular Endothelial Growth Factor Receptor (VEGFR), and fms-related tyrosine kinase 3 ( Flt3), whereas cytoplasmic type tyrosine kinase proteins are

Biomolecular and Health Science Journal 
the janus kinase group (JAK), the Src kinase group (SFKs), and the ABL kinase. ${ }^{4,5}$

JAK plays an important role in hematopoietic cells cytokine signaling. JAK is involved in signal transduction in type 1 cytokine receptors, namely for Interlekuin (IL) -2, IL-3, IL-4, IL-5, IL-6, IL-7, IL-13, Granulocyte Macrophage-Colony Stimulating Factor (GM-CSF), Growth Hormone (GH), Prolactin (PRL), Erythropoietin (EPO), and Thrombopoietin (TPO), as well as type 2 receptors, namely Interferon (IFN) $-\alpha$, IFN- $\beta$, and IFN- $\gamma .{ }^{6}$

There are currently 4 JAK groups, namely JAK1, JAK2, JAK3 and Tyk2. ${ }^{7}$ JAK is divided into 7 areas called domains 1-7 JAK homology (JH) (figure 1). The $\mathrm{JH} 1$ domain is catalytic phosphotyrosine kinase, the $\mathrm{JH} 2$ domain functions as a pseudokinase domain and is believed to be able regulate both basal JAK activity and to activate catalytic induced receptor function. Interestingly, because of the absence of ligand bond, interaction of $\mathrm{JH} 1$ and $\mathrm{JH} 2$ domains will manifest an autoinhibition device, means if there is a change in this area there will be autoinhibition dysregulation. The JH3JH4 domain is a homologous domain with the Srchomology-2 (SH2) domain, but not linked to phosphotyrosine residues. The JH5-JH7 is the FERM domain (Four-point-one, Ezrin, Radxin, Moesin), which mediates JAK and other proteins. ${ }^{6,8,9}$

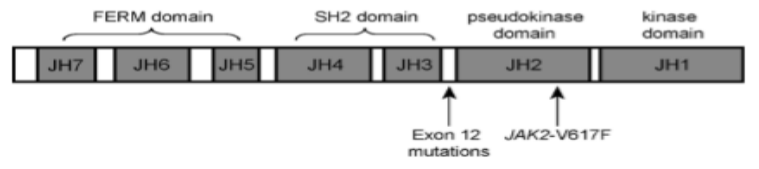

Figure 1. Domain of Janus homolog JAK family of kinases.

Under normal conditions, activation of JAK-STAT (signal transducers and activators) is initiated by ligand bonds and receptors which then induce receptor dimerization, which results in auto-transfusion of JAK2related receptors. The part of the phosphorylated receptor will then act as a place for the recruitment and phosphorylation of signal-sending molecules, that triggers the encoding process through STAT resulting in dimerization and translocation of the STAT molecule into the nucleus where the interaction with specific regulatory elements will induce target gene transcription. ${ }^{10}$

The number and duration of JAK2 encoding are strictly regulated by several mechanisms to prevent unnecessary encoding which can lead to uncontrolled proliferation and transformation towards malignancy. The mechanisms include JAK2 direct dephosphorylation by certain protein tyrosine phosphatase (such as SHP-1), proteolytic degradation of JAK2 by binding in with Suppressors of Cytokine Signaling (SOCS) and inhibition of STAT binding DNA by Protein Inhibitors of Activated STAT (PIAS). ${ }^{10,11}$

\section{JAK2 Mutation}

In the context of hematopoiesis, encoding JAK-STAT is essential for definitive hematopoesis and cytokine response in myeloid progenitors, therefore it is not surprising if mutations from molecules related to JAKSTAT encoding or its regulators will be related to hematological malignancies. ${ }^{4,12}$

JAK2 $2617 \mathrm{~F}$ is an exon $14 \mathrm{G}$ to $\mathrm{T}$ somatic mutation in JAK2 which resulted in substitution of valine to phenylalanine at codon 617. JAK2V617F association with MPD, including PV, ET, and PMF, was first reported in 2005. JAK2V617F is not found in lymphoid disorders, solid tumors, or secondary myeloproliferation. ${ }^{4}$ This mutation cause signal transmission to become more sensitive and efficient than EPO receptor, TPO receptor, and G-CSF receptor to haematopoietic cells and cause loss of autoinhibition of JAK2 activity. ${ }^{13,14}$

Detection of JAK2V617F mutation can be done with alel-specific PCR that use neutrophil. A positive test result strongly indicated MPD because it is usually negative in all normal individuals and hematological abnormalities such as secondary erythrocytosis, reactive thrombocytosis, leukemoid reactions, and secondary myelofibrosis. This test is also negative for normal $\mathrm{B}$ lymphocytes, normal $\mathrm{T}$ lymphocytes, lymphoma, sarcoma, and solid tumors. In addition, this test is also usually negative for chronic myeloid leukemia, acute myeloid leukemia (except those who develop from MPD), and myelodysplastic syndrome. In addition, the test can also be useful as a sensitive marker of MPD detection as a basic etiology of splanchnic venous thrombosis affecting the spleen vein, portal vein, and hepatic vein. ${ }^{13}$

\section{Impact on Diagnosis}

PV patients often show symptoms like headache, pruritus, vaso-occlusive symptoms such as tinnitus, paresthesia, and erythromelalgia. Splenomegaly is seen in some patients with a size that is directly proportional to the stage of the disease. Typical characteristics of PV are high hematocrit, erythrocytosis, and increased hemoglobin levels. Bone marrow biopsy shows hypersellularity with increased proliferation of three lines (panmielosis), especially erythroid, granulocytic proliferation, and megakariocytic. ${ }^{15}$

The actual erythrocyte volume status is not enough if only described by hemoglobin levels and hematocrit alone. Increased hemoglobin and hematocrit levels can show a true polycythemia (increased erythrocyte volume) or apparent polycythemia (not associated with increased erythrocyte volume). True polycythemia includes PV and non-clonal erythrocytosis, namely secondary polycythemia (mediated by EPO). While apparent polycythemia is a result of plasma volume 
decrease (relative polycythemia). Inapparent PV used to describe PV patients who have an increase in erythrocyte volume but are obscured by normal hematocrit values due to an increase in plasma volume. Therefore, in suspected PV patients, secondary polycythemia and apparent polycythemia must be excluded. ${ }^{4}$

In PV patients, erythroid progenitors can grow without the addition of exogenous erythropoetin, which is the initial etiological factors in cytokine regulation. JAK2 mutation was finally approved to be the main etiology and can be found in $95 \%$ PV patients, which led to WHO diagnostic criteria revision (table 1). ${ }^{16}$

Although, most PV patients have positive JAK2V617F, specific alele test shows the opposite. Scott and colleagues looked for alternative aleles that might result in JAK-STAT activation by analyzing PV patients with negative JAK2V617F in all exons from JAK1, JAK2, JAK3, TYK2, STAT5A, and STAT5B. Genomic analysis identified four new somatic mutations in exon 12 from JAK2; In vitro colony tests showed that there were growths in colonies that had JAK2 exon 12 mutations that were not EPO dependent. ${ }^{3,11}$

ET is clinically characterized by thrombosis, headache, dizziness, syncope, acrosianosis, and changes in vision. Mild splenomegaly occurs in $40 \%$ of patients, but $25-35 \%$ of cases are asymptomatic and are diagnosed accidentally. Based on laboratory examination, ET is characterized by thrombocytosis and megacryocyte hyperplasia in bone marrow examination, without accompanied by erythrocytosis and leukoeritroblastosis. Megakariocyte precursors showed hypersentivity to thrombopoetin with JAK2V617F as the main molecular lesion that appeared with a frequency of $50 \%{ }^{15}$

ET is the only MPD that does not have a specific phenotype. Because single thrombocytosis can be an early clinical manifestation of PV, PMF or CML. ${ }^{2}$

Table 1. Comparison and revision of 2008 WHO diagnostic criteria Polycythemia Vera

\begin{tabular}{|c|c|}
\hline $\begin{array}{c}\text { British Committee for Standards in } \\
\text { Haematology Guidelines on Polycythaemia }\end{array}$ & WHO 2008 Criteria $^{4}$ \\
\hline - Major criteria & - Major criteria \\
\hline $\begin{array}{l}\text { A1: Raised red cell mass }(>25 \% \text { above mean normal } \\
\text { predicted value) or Hct } \geq 0.60 \text { males; } \geq 0.56 \\
\text { females }\end{array}$ & $\begin{array}{l}\text { 1. Hemoglobin }>18.5 \mathrm{~g} / \mathrm{dL} \text { in men, }>16.5 \mathrm{~g} / \mathrm{dL} \text { in } \\
\text { women; or evidence of increased red blood cell } \\
\text { volume }\end{array}$ \\
\hline $\begin{array}{l}\text { A2: Absence of cause for secondary erythrocytosis } \\
\text { (consider possibility of dual pathology) }\end{array}$ & $\begin{array}{l}\text { 2. Presence of JAK2V617F or other clonal markers } \\
\text { such as JAK2 ekson } 12 \text {. }\end{array}$ \\
\hline \multicolumn{2}{|l|}{ A3: Palpable splenomegaly } \\
\hline \multicolumn{2}{|l|}{$\begin{array}{l}\text { A4: Clonality marker, i.e. acquired abnormal marrow } \\
\text { karyotype }\end{array}$} \\
\hline - Minor criteria & - Minor criteria \\
\hline B1: Thrombocytosis (platelet count $>400 \times 10^{9} / 1$ ) & $\begin{array}{l}\text { 1. Bone marrow biopsy shows hypercellularity with } \\
\text { increased proliferation of three lines (panmyelosis), } \\
\text { especially erythroid, granulocytic and } \\
\text { megakaryocytic proliferation }\end{array}$ \\
\hline $\begin{array}{l}\text { B2: Neutrophil leucocytosis (neutrophil count } \\
>10 \times 10^{9} / 1 \text { in non-smokers; }>12 \cdot 5 \times 10^{9} / 1 \text { in } \\
\text { smokers) }\end{array}$ & 2. Normal or subnormal serum erythropoietin level \\
\hline $\begin{array}{l}\text { B3: Splenomegaly (demonstrated on } \\
\text { isotope/ultrasound scanning) }\end{array}$ & 3. In vitro endogenous erythroid colony formation \\
\hline \multicolumn{2}{|l|}{$\begin{array}{l}\text { B4: Characteristic BFU-E growth or reduced serum } \\
\text { erythropoietin } \S\end{array}$} \\
\hline $\begin{array}{l}\text { Diagnosis } \\
\text { - } \mathrm{A} 1+\mathrm{A} 2+\mathrm{A} 3 / \mathrm{A} 4, \text { or } \\
\text { - } \mathrm{A} 1+\mathrm{A} 2+2 \mathrm{~B}\end{array}$ & $\begin{array}{l}\text { Diagnosis } \\
\text { - } 2 \text { major criteria }+1 \text { minor criteria, or } \\
\text { - } 1 \text { major criteria }+2 \text { minor criteria }\end{array}$ \\
\hline
\end{tabular}

In a survey of 732 patients with platelet counts greater than $500,000 / \mu \mathrm{L}$, only 89 patients $(12.3 \%)$ had clonal disorders and 40 people had ET. Although platelet counts are not the main standard for the ET diagnosis, there is a significance accuracy betwen the higher platelet count. 
The JAK2V617F mutation has made it possible to reduce the platelet number threshold for TE diagnosis from $600 \times 109 / \mathrm{L}$ to $450 \times 109 / \mathrm{L}$, means the WHO 2001 diagnosis criteria for TE were revised by WHO 2008 (Table 2). 2, 16 
Table 2. Comparison and revision of 2008 WHO diagnostic criteria of Essential Thromobocytosis

\begin{tabular}{|c|c|}
\hline UK MPD group $^{8}$ & WHO 2008 Criteria $^{4}$ \\
\hline $\begin{array}{l}\text { 1. Thrombocyte count > } \\
600 \times 10^{9} / \mathrm{L}\end{array}$ & $\begin{array}{l}\text { 1. Thrombocyte count } \\
\text { consistently above } 450 \\
\text { x } 10^{9} / \mathrm{L}\end{array}$ \\
\hline $\begin{array}{l}\text { 2. Absent evidence of } \\
\text { overt polycythemia/ } \\
\text { polycythemia covered } \\
\text { by iron deficiency }\end{array}$ & $\begin{array}{l}\text { 2. Bone marrow biopsy } \\
\text { shows proliferation of } \\
\text { megakaryocyte line } \\
\text { with increased number } \\
\text { of mature and enlarged } \\
\text { megakaryocyte }\end{array}$ \\
\hline $\begin{array}{l}\text { 3. Absence } \\
\text { Philadelphia } \\
\text { chromosome }\end{array}$ & $\begin{array}{l}\text { 3. Does not meet WHO } \\
\text { criteria for PV, PMF, } \\
\text { CML and MDS or } \\
\text { other myeloid } \\
\text { neoplasia }\end{array}$ \\
\hline $\begin{array}{l}\text { 4. Absence of signs of } \\
\text { myelodysplasia or } \\
\text { myelofibrosis evident } \\
\text { from } \text { peripheral } \\
\text { smears or } \text { bone } \\
\text { marrow }\end{array}$ & $\begin{array}{l}\text { 4. Presence of } \\
\text { JAK2V617F or other } \\
\text { clonal markers; or } \\
\text { absence of clonal } \\
\text { markers and evidence } \\
\text { of reactive } \\
\text { thrombocytosis } \\
\end{array}$ \\
\hline \multicolumn{2}{|l|}{$\begin{array}{l}\text { 5. Absence of causes of } \\
\text { reactive } \\
\text { thromobcytosis }\end{array}$} \\
\hline Diagnosis & Diagnosis \\
\hline $\begin{array}{l}\text { Requires the five citeria } \\
\text { above }\end{array}$ & $\begin{array}{l}\text { Requires the four citeria } \\
\text { above }\end{array}$ \\
\hline
\end{tabular}

People with PMF can show symptoms of tightness, fatigue, night sweats, cachexia, fever, and bleeding. More than $90 \%$ patients have splenomegaly. However, $30 \%$ of patients are asymptomatic. People with PMF can have anemia, leukocytosis and/or thrombocytosis. Early stage PMF, "Pre-fibrotic stage" is difficult to diagnose because the initial symptoms of thrombosis are similar to ET. Typical characteristics of myelofibrosis, whether coming from pure PMF or advanced stages of post $\mathrm{TE} / \mathrm{PV}$, are accumulation of reticulin fibers in the bone marrow accompanied by hypercellularity with granulopoesis expansion, decreased erythropoesis, and megakaryocyte hyperplasia. PMF shares aetiological factors with PV and ET, namely JAK2V167F in 50\% of patients, so the WHO 2001 diagnosis criteria for PMF have also been revised by WHO (table 3 ). ${ }^{16}$

JAK2V617F Alel Load

PV, ET, and PMF often share similar disease characteristics. Although erythrocytosis is a marker of $\mathrm{PV}$, the three disorders can show thrombocytosis. Splenomegaly is very common in PMF, as much as $30 \%$ can also appear in TE and PV patients. Bone marrow fibrosis is a typical sign of PMF, but TE and PV can also experience reticulin fiber accumulation. Both TE and PV have a tendency to develop into myelofibrosis and all above can turn into acute myeloid leukemia.

The most interesting question regarding the discovery of this mutation is how a mutation can cause three different diseases. Although it has not been fully answered, clinical, biological and pathological data have produced three hypotheses. First, the gene dose hypothesis, postulated a relationship between disease phenotype and the proportion of mutant JAK2V617F aleles, introducing a concept of alele load (ratio between JAK2 mutations and wild JAK2 in hematopoiesis cells). The second is about pre-JAK2 phase dimheterozigotana additional mutations and inherited aleles affecting the appearance of clonal hematopoiesis before the presence of JAK2V617F. The third hypothesis is host genetic factors that can contribute to the diversity of phenotypes. $^{17}$

Several studies have shown that alel loads are associated with haematological characteristics and late clinical conditions in MPD patients, although half are agreed that alel mutation is not the only basic mechanism for pleiotropic MPD. The burden of the JAK2 mutation has an influence on the disease phenotype through increasing the activation level of the JAK-STAT encoding pathway. This is shown by the difference JAK2V617F alele amount load on granulocytes with MPD. The highest level found in PV and the lowest level were found in ET, while medium level found in PMF patients. $^{18}$

In the initial study of JAK2V617F aleles in PV, ET and PMF, it was seen that although most MPD patients had heterozygous JAK2V617F aleles, some populations of PV patients were homozygous. The mechanism of homozygosity for JAK2V617F is the result of recombination of mitosis and duplication MPD alele mutants on chromosome 9 p24 locus, known as acquired uniparental disomy (UPD). Homozygous JAK2V617F is found in almost all patients with PV. Conversely, homozygous JAK2V617F mutations are rarely seen in ET, where hematopoietic colonies that often develop are wild type or heterozygous JAK2. ${ }^{19}$

The largest study comparing homozygous JAK2V617F and heterozygotes JAK2V617F in PV and ET patients involved $323 \mathrm{PV}$ patients $(67.8 \%$ heterozygous and $32.2 \%$ homozygous) and 639 ET patients $(40.2 \%$ wild type, $57.6 \%$ heterozygous, and 2,2\% homozygous).

In both diseases, homozygous conditions are associated with erythropoesis and stimulated myelopoesis, low platelet counts, high incidence of 
Table 3. Comparison and revision of 2008 WHO diagnostic criteria of Primary Myelofibrosis

\begin{tabular}{c}
$\begin{array}{c}\text { Italian Consensus on Diagnostic Criteria for } \\
\text { Myeloibrosis }\end{array}$ \\
\hline Necessary criteria
\end{tabular}

$\frac{\text { Necessary criteria }}{\text { 1. Diffuse bone marrow fibrosis }}$

2. Absence of Philadhelpia chromosome

\section{WHO 2008 Criteria $^{4}$}

Major criteria

1. Presence of megakaryocytic proliferation accompanied by reticuline fibrosis; if not accompanied by reticuline fibrosis, megakaryocytic changes must be followed by increased bone marrow cellularity in the form of granulocytic proliferation and sometimes reduced erythropoiesis

2. Does not meet WHO criteria for PV, CML and MDS or other myeloid neoplasia

3. Presence of JAK2V617F or other clonal markers; or if clonal marker is absent, no evidence of bone marrow fibrosis due to inflammation or other neoplasia

\begin{tabular}{l|l}
\hline Optional criteria & Minor criteria \\
\hline 1. Any grade of splenomegaly & 1. Myeloid metaplasia \\
\hline $\begin{array}{l}\text { 2. Anisopoikilocytosis with tear drop erythrocytes } \\
\text { 3. Presence of immature myeloid cells in peripheral } \\
\text { circulation }\end{array}$ & 2. Increased serum level of lactate dehydrogenase \\
\hline 4. Presence of erythroblasts in peripheral circulation & 4. Splenomegaly \\
\hline $\begin{array}{l}\text { 5. Presence of megakaryoblast lusters and abnormal } \\
\text { megakaryocytes in bone marrow }\end{array}$ & \\
\hline 6. Myeloid metaplasia & Diagnosis \\
\hline Diagnosis & $\bullet 3$ major criteria+ 2 minor criteria \\
\hline $\begin{array}{l}\text { - } 2 \text { necessary criteria, with an addition of: } \\
\text { 4 optional criteria if splenomegaly is absent }\end{array}$ & \\
\hline
\end{tabular}

splenomegaly and a greater proportion in need of cytoreductive therapy. Homozygous PV patients also have higher incidence of pruritus $(42 \%)$. The rate of thrombosis is not increased in homozygous PV compared to heterozygous one. ${ }^{18}$

In contrast, thrombosis events were more common in homozygous ET patients, with a hazard ratio of 3.97

times higher than those with ET wild type JAK2. The relationship of homozygosity with cardiovascular events also seen in multivariate analysis where other factors that affected were age $>60$ years, history of previous thrombosis, and leukocytosis above $8.7 \times 109 /$ L. $^{18}$

Furthermore, studies from Vannucchi and Tefferi showed that the number of PV patients who experienced

fibrosis transformation was significantly greater in the homozygous group than in the heterozygote group, ie $23 \%$ compared to $2 \%, \mathrm{p}=0.009$, in the Mayo study (Tefferi et al. 2006) and $11.5 \%$ compared to $1.4 \%$, $\mathrm{p}<0.001$, in the Italian study. The risk of having fibrosis transformation was also significantly higher among homozygous ET patients (homozygous 14.3\%, heterozygous $4.7 \%$, and wild type $1.6 \%$, p<0.001). ${ }^{20}$

The relationship between mutant alele load and the risk of mielofibrosis also has been confirmed by multivariate analysis in PV patients. Conversely the risk of acute leukemia development is not significantly associated with mutant alele load. Overall. These data indicate that the JAK2V617F alele load is related to the myeloproliferation range and the risk of mielofibrosis evolution in both PV and ET. ${ }^{17}$

\section{Conclusion}

JAK plays an important role in cytokine signaling of hematopoietic cells. Mutation related to JAK-STAT encoding or regulators will be related to hematological malignancies. JAK2 $2617 \mathrm{~F}$ is a somatic $\mathrm{G}$ to $\mathrm{T}$ exon 14 mutation which results in substitution of valine to phenylalanine at codon 617 . JAK2V617F mutations have the ability to transmit signals from EPO, TPO, and GCSF receptors in haematopoietic cells more efficiently and cause loss of autoinhibition of JAK2 activity. The relationship between JAK2V617F and MPD, including $\mathrm{PV}, \mathrm{TE}$, and PMF, was first reported in 2005. Various evidences that show association between PV and JAK2 mutation led to a revision of WHO diagnostic criteria for PV that include screening of JAK2 mutations. Several studies have shown that JAK2 mutation alele load is related to hematological characteristics and prognosis of MPD patients. 


\section{Conflict of Interest}

The author stated there is no conflict of interest.

\section{References}

1. Kiladjian JJ. The Spectrum of JAK2-Positive Myeloproliferative Neoplasms. Hematology American Society of Hematology Education Program. 2012; 2012: 561-6.

2. Spivak JL and Silver RT. The Revised World Health Organization Diagnostic Criteria for Polycythemia Vera, Essential Thrombocytosis, and Primary Myelofibrosis: an Alternative Proposal. Blood. 2008; 112: 231-9.

3. Latif N, Guthrie $\mathrm{T}$ and Rana F. JAK2 Gene Mutation: Impact on Pathogenesis, Classification, and Management of Myeloproliferative Neoplasms. 2010, p.109-14.

4. Tefferi A. JAK2 Mutations and Clinical Practice in Myeloproliferative Neoplasm. Cancer Journal. 2007; 13: 366-71.

5. Sansone $\mathrm{P}$ and Bromberg J. Targeting the Interleukin6/JAK/STAT Pathway in Human Malignancies. Journal of clinical oncology : official journal of the American Society of Clinical Oncology. 2012; 30: 1005-14.

6. Haan C, Kreis S, Margue C and Behrmann I. JAKs And Cytokine Receptors--An Intimate Relationship. Biochem Pharmacol. 2006; 72: 1538-46.

7. O'Shea JJ. JAKs, STATs, Cytokine Signal Transduction, and Immunoregulation: Are We There Yet? Immunity. 1997; 7: 1-11.

8. McLornan D, Percy M and McMullin MF. JAK2 V617F: A Single Mutation in the Myeloproliferative Group of Disorders. The Ulster medical journal. 2006; 75: 112-9.

9. Seavey MM and Dobrzanski P. The Many Faces of Janus Kinase. Biochem Pharmacol. 2012; 83: 1136-45.

10. Bromberg J. STAT Proteins and Oncogenesis. The Journal of clinical investigation. 2002; 109: 1139-42.

11. Tefferi A and Vainchenker W. Myeloproliferative Neoplasms: Molecular Pathophysiology, Essential Clinical Understanding, and Treatment Strategies. Journal of clinical oncology : official journal of the American Society of Clinical Oncology. 2011; 29: 573-82.

12. Hou SX, Zheng Z, Chen $X$ and Perrimon N. The JAK/STAT Pathway in Model Organisms: Emerging Roles in Cell Movement. Developmental cell. 2002; 3: 765-78.

13. Agarwal MB. Clinical Applications of Molecular Haematology: JAK2 in Myeloproliferative Disorders. The Journal of the Association of Physicians of India. 2007; 55: 507-10.

14. Hsu HC. Pathogenetic Role of JAK2 V617F Mutation in Chronic Myeloproliferative Disorders. Journal of the Chinese Medical Association : JCMA. 2007; 70: 89-93.
15. James C, Ugo V, Le Couedic JP, et al. A Unique Clonal JAK2 Mutation Leading to Constitutive Signalling Causes Polycythaemia Vera. Nature. 2005; 434: 1144-8.

16. Tefferi A, Lasho TL, Schwager SM, et al. The Clinical Phenotype of Wild-Type, Heterozygous, and Homozygous JAK2V617F in Polycythemia Vera. Cancer. 2006; 106: 631-5.

17. Passamonti F and Rumi E. Clinical Relevance of JAK2 (V617F) Mutant Allele Burden. Haematologica. 2009; 94: 7-10.

18. Vannucchi AM, Pieri L and Guglielmelli P. JAK2 Allele Burden in the Myeloproliferative Neoplasms: Effects on Phenotype, Prognosis and Change with Treatment. Therapeutic Advances in Hematology. 2011; 2: 21-32.

19. Tiedt R, Hao-Shen H, Sobas MA, et al. Ratio of Mutant JAK2-V617F to Wild-Type JAK2 Determines The MPD Phenotypes in Transgenic Mice. Blood. 2008; 111: 393140.

20. Vannucchi AM, Antonioli E, Guglielmelli P, et al. Clinical Profile of Homozygous JAK2 V617F Mutation in Patients with Polycythemia Vera or Essential Thrombocythemia. Blood. 2007; 110: 840-6. 